\title{
Sepsis-3 y las nuevas definiciones, ¿es tiempo de abandonar SIRS?
}

\section{Sepsis-3 new definitions, is it time to quit SIRS?}

Correspondencia Germán Málaga

german.malaga@upch.pe

\section{Recibido: 16/08/2016}

Arbitrado por pares

Aprobado: 07/09/2016

Citar como: Neira-Sanchez ER, Málaga G. Sepsis-3 y las nuevas definiciones, ¿es tiempo de abandonar SIRS? Acta Med Peru. 2016;33(3):217-22

\author{
Elsa R Neira-Sanchez 1,2,3, Germán Málaga 1,3,a \\ 1 Departamento de Medicina, Hospital Nacional Cayetano Heredia. Lima, Perú. \\ 2 Universidad Peruana Cayetano Heredia. Lima, Perú. \\ 3 Conocimiento y Evidencia CONEVID, Universidad Peruana Cayetano Heredia. Lima, Perú.
}

\section{RESUMEN}

Hace más de 20 años se publicó la primera definición de sepsis basado en el concepto de Respuesta Inflamatoria Sistémica (SIRS), definición que utilizamos hasta la actualidad. Recientemente, el Grupo de Trabajo de las Definiciones de Sepsis (Sepsis Definitions Task Force) ha publicado un consenso con las definiciones actualizadas de sepsis y shock séptico (SEPSIS-3) que utiliza un nuevo concepto de sepsis como disfunción orgánica potencialmente mortal causada por una respuesta disregulada del huésped a la infección, utilizando el sistema de puntuación SOFA (Sequential Organ Failure Assessment), y propugna se abandonen los antiguos criterios. Realizamos una revisión de los antecedentes de esta nueva propuesta de definición, las definiciones planteadas por este nuevo consenso, comparando estas nuevas definiciones con las antiguas, limitaciones y evaluamos su aplicabilidad en la práctica clínica.

Palabras clave: Sepsis; Consenso; Diagnóstico; Infección (fuente: DeCS BIREME).

\section{ABSTRACT}

More than 20 years ago, the first definition of sepsis based on the concept of systemic inflammatory response syndrome (SIRS), was published, and this is the definition we have been currently using until now. Recently, the Sepsis Definitions Task Force has published a consensus statement with the updated definitions of sepsis and septic shock (sepsis-3), and introducing a new concept of sepsis as a life-threatening organ dysfunction caused by a dysregulated host response to infection, using the SOFA scoring system (Sequential Organ Failure Assessment), and calls for abandoning the old criteria. We review the background for this new proposed definition, the definitions proposed by this new consensus, and we compare the new definitions with respect to the old ones, aiming at determining their limitations and evaluating their applicability in clinical practice.

Key words: Sepsis; Consensus; Diagnosis; Infection (source: MeSH NLM). 


\section{INTRODUCCIÓN}

El término sepsis fue introducido por Hipócrates en el siglo IV a.C. como un proceso por el cual la carne se descompone y las heridas se infectan ${ }^{[1]}$. Sin embargo, ante la inexistencia de una definición o criterios diagnósticos uniformes, en 1992 se publicó el consenso del American College of Chest Physicians y la Society of Critical Care Medicine Consensus Conference para uniformizar los criterios diagnósticos y lograr la oportuna "sospecha diagnóstica", criterios que provocaron controversia desde el momento en que fueron presentados ${ }^{[2]}$. En los últimos años, los avances en los conocimientos de la fisiopatología, han demostrado que las infecciones provocan una respuesta compleja y variable del huésped, con mecanismos proinflamatorios y antiinflamatorios que pueden contribuir por un lado a la eliminación de la infección y la recuperación de los tejidos y por otro lado a la injuria de los órganos e infecciones secundarias; la respuesta va a depender del patógeno causal (carga y virulencia) y de las características genéticas y enfermedades coexistentes del huésped ${ }^{[3,4]}$.

\section{SEPSIS-1}

En el año 1992 se publicó el primer consenso del American College of Chest Physicians/Society of Critical Care Medicine ${ }^{[2]}$ que estableció el concepto de respuesta inflamatoria sistémica (SIRS, por sus siglas en inglés) como la respuesta a una variedad de insultos clínicos severos y que se manifiesta como la presencia de dos o más de los siguientes hallazgos: (1) temperatura $>38^{\circ} \mathrm{C}$ $0<36^{\circ} \mathrm{C}_{i}$ (2) frecuencia cardíaca $>90$ latidos por minuto; (3) frecuencia respiratoria $>20$ respiraciones por minuto o $\mathrm{PaCO}_{2}<32$ $\mathrm{mmHg} ; \mathrm{y}(4)$ recuento de leucocitos $>12000 \mathrm{cel} / \mu \mathrm{l},<4000 \mathrm{cel} / \mu \mathrm{l}$, $0>10 \%$ de formas inmaduras, y desde ese momento, el concepto de SIRS fue adoptado por clínicos e investigadores. Este mismo consenso define "sepsis" como la respuesta inflamatoria sistémica asociada a una infección. Del mismo modo, este consenso establece que la sepsis conlleva otras patologías por una continuidad de severidad clínica y fisiopatológica: hipotensión inducida por sepsis, sepsis severa, shock séptico y síndrome de disfunción de múltiples órganos (MODS, por sus siglas en inglés). Desde entonces, estas definiciones se han utilizado en la práctica clínica y en la investigación como criterios de definición en los ensayos clínicos, y hasta ahora las había posicionado como inamovibles. El espíritu de este consenso, fue el de proveer una herramienta o criterios de diagnóstico que permitieran hacer el diagnóstico de sepsis de manera precoz, como una de las pocas herramientas efectivas para aplacar su aún elevada mortalidad.

\section{SEPSIS-2}

En el año 2001, el grupo de expertos del Society of Critical Care Medicine (SCCM), la European Society of Intensive Care Medicine (ESICM), la American College of Chest Physicians (ACCP), la American Thoracic Society (ATS), y la Surgical Infection Society (SIS) ${ }^{[5]}$ recomendó que las definiciones de sepsis, sepsis severa y shock séptico del año 1992 deberían mantenerse, este consenso expandió la lista de criterios diagnósticos, aumentando parámetros generales, parámetros hemodinámicos, parámetros inflamatorios y parámetros de perfusión tisular, pero no sugirió cambios en las definiciones por ausencia de evidencia (Tabla 1).

\section{¿ES NECESARIA UNA NUEVA DEFINICIÓN DE SEPSIS? CUESTIONAMIENTOS:}

\section{Especificidad y sensibilidad}

La definición de sepsis basada en los criterios de SIRS ha sido ampliamente criticada por su alta sensibilidad, y es que con ellos, cerca del $90 \%$ de los pacientes admitidos a una Unidad de Cuidados Intensivos ( $\mathrm{UCl}$ ) cumplen con la definición de sepsis $[6,7]$. Sin embargo, estos criterios tienen una baja especificidad, como la reportada en el estudio de Lai NA et al., que encontró que los criterios de SIRS para predecir una infección confirmada microbiológicamente tienen una sensibilidad de 70,6\%, especificidad de $37,5 \%$, valor predictivo positivo de $63,7 \%$ y valor predictivo negativo de $45,1 \%{ }^{[7]}$. Estos valores son similares a los reportados anteriormente por Jaimes $\mathrm{F}$ et al. ${ }^{[8]}$ en relación a los criterios de SIRS, quien describió sensibilidad de $69 \%$, especificidad de $35 \%$, valor predictivo positivo de $90 \%$ y valor predictivo negativo de $12 \%{ }^{[9,10]}$, esta baja especificidad se atribuye a que esta definición incluye una respuesta inflamatoria sistémica que se presenta en diversas patologías infecciosas y no infecciosas, como infarto agudo de miocardio, pancreatitis, quemaduras, entre otras que son procesos inflamatorios estériles. Por otro lado, si bien es cierto que, por definición, la sepsis es una respuesta inflamatoria con infección, la presencia de infección por sí sola no implica la presencia de sepsis, tal como se da en la colonización.

Adicionalmente, con relación al diagnóstico de sepsis severa, recientemente el estudio de Kaukonen KM et al. ${ }^{[1]}$ utilizando los criterios establecidos para su diagnóstico en las primeras 24 horas de la admisión a $172 \mathrm{UCls}$, encontró que se deja de diagnosticar un paciente de cada ocho con sepsis severa. De esta manera, este estudio concluyó que la definición de sepsis severa tenía limitada sensibilidad, baja especificidad y cuestiona la validez aparente de construcción de la definición de sepsis severa, incluso como una definición a la que le falta al definir el riesgo de muerte en estos pacientes.

\section{Cambios en la definición}

A los cuestionamientos mencionados, se agrega el hecho de que en el "Simposio Merinoff, Foro Internacional de Sepsis", se propuso una nueva definición, "sepsis es una afección potencialmente mortal que se produce cuando la respuesta del cuerpo a una infección ataca a sus propios tejidos y órganos", y a la vez propone una definición molecular "las moléculas derivadas del huésped y productos extraños de la infección convergen sobre los mecanismos moleculares que producen una activación desequilibrada de la inmunidad innata. Las moléculas extrañas y endógenas interactúan con los receptores de reconocimiento de los patógenos expresados sobre o en las células del sistema 
inmune. La activación de los receptores de reconocimiento de los patógenos culmina en la liberación de mediadores inmunes que producen los signos y síntomas de la sepsis" [12].

De esta manera, se pone en relieve, que la sepsis, más que una respuesta inflamatoria con infección, es un síndrome difícil de definir por los diversos procesos fisiopatológicos e inmunológicos involucrados y muchos de ellos aún inciertos y con diversa presentación clínica la que puede variar dependiendo de la edad del paciente, sus enfermedades prexistentes, estado inmunológico, comorbilidades y medicación.

Otro problema asociado, es que los términos sepsis y sepsis severa se tienden a usar indistintamente, y esta es una confusión crítica cuando nos referimos a sepsis severa, con la que estamos tratando de definir a un paciente que necesita cuidado médico estricto, con monitoreo hemodinámico, pues estamos implicando disfunción orgánica, elevación de lactato, alteraciones en el medio interno, trastorno del sensorio y otras condiciones que ponen en riesgo la vida del paciente, pues el no reconocimiento temprano, permite que la sepsis severa progrese con shock séptico, la falla orgánica múltiple y la muerte. La sepsis es la primera causa de muerte por infección a pesar de los avances en la medicina moderna, incluyendo vacunas, antibióticos y cuidados críticos agudos, millones de personas mueren por sepsis cada año en el mundo, sólo en Estados Unidos aproximadamente 215000 personas fallecen cada año ${ }^{[12]}$.

\section{Ausencia de correlación con mortalidad}

La sepsis, sepsis severa y shock séptico son condiciones que conllevan alta morbilidad y mortalidad, por esto, es deseable para los médicos de atención primaria y de emergencias, poder definir "sepsis" como se definen los criterios de otras patologías como la endocarditis bacteriana, con criterios de alta sensibilidad y sobretodo alta especificidad y que permitan una sospecha temprana, ya que ante el diagnóstico de sepsis, nuestro objetivo inmediato es determinar la severidad del cuadro de sepsis y el grado de compromiso multiorgánico para iniciar a la brevedad posible el manejo antibiótico y soporte hemodinámico en presencia de hipotensión o shock y determinar el ingreso a una UCI para prevenir la falla multiorgánica y optimizar los resultados clínicos.

Tabla 1. Criterios diagnósticos de sepsis *

\section{SEPSIS}

Infección documentada o sospechada y uno de los siguientes parámetros:

Parámetros generales

Fiebre (temperatura $>38,3^{\circ} \mathrm{C}$ )

Hipotermia (Temperatura $<36^{\circ} \mathrm{C}$ )

Frecuencia cardíaca > 90 latidos/minuto 02 DS por encima del valor normal para la edad

Taquipnea $>30$ respiraciones/minuto

Alteración del estado mental

Edema significativo o balance de fluidos positivo (> $20 \mathrm{~mL} / \mathrm{kg}$ en 24 horas)

Hiperglicemia (glucosa plasmática $>110 \mathrm{mg} / \mathrm{dL}$ ) en ausencia de diabetes

Parámetros inflamatorios

Leucocitosis (recuento de glóbulos blancos $>12000 / \mu \mathrm{L}$ )

Leucopenia (recuento de glóbulos blancos $<4000 / \mu \mathrm{L}$ )

Recuento de glóbulos blancos normal con más del $10 \%$ de formas inmaduras

Proteína $C$ reactiva en plasma> 2 DS sobre el valor normal

Procalcitonina en plasma > 2 DS sobre el valor normal

Parámetros hemodinámicos

Hipotensión arterial (PAS $<90 \mathrm{mmHg}, \mathrm{PAM}<70 \mathrm{mmHg}$ o disminución PAS $>40 \mathrm{mHg}$ en adultos $0<2$ DS debajo de lo normal para la edad)

Saturación venosa mixta de oxígeno $>70 \%$

Índice cardíaco $>3,5 \mathrm{~L} / \mathrm{min} / \mathrm{m}^{2}$

Parámetros de disfunción orgánica

Hipoxemia arterial $\left(\mathrm{PaO}_{2} / \mathrm{FiO}_{2}<300\right)$

Oliguria aguda (gasto urinario $<0,5 \mathrm{ml} / \mathrm{kg} / \mathrm{h} 045 \mathrm{~mm} / \mathrm{L}$ por lo menos 2 horas)

Incremento de la creatinina $\geq 0,5 \mathrm{mg} / \mathrm{dL}$

Anormalidades de la coagulación: INR > 1,5 0 TTP activado $>60$ segundos)

lleo (ausencia de ruidos hidroaéreos)

Trombocitopenia (Recuento de plaquetas $<100000 / \mu \mathrm{L}$ )

Hiperbilirrubinemia (Bilirrubina total en plasma $>4 \mathrm{mg} / \mathrm{dL}$ )

Parámetros de perfusión tisular

Hiperlactatemia (>3 mmol/L)

Disminución del llenado capilar o moteado

PAS: presión arterial sistólica, PAM: presión arterial media, $\mathrm{PaO}_{2}$ : presión arterial de oxígeno, FiO : fracción inspirada de oxígeno * Adaptado de Levy et al. ${ }^{55]}$ 
Tabla 2. Puntuación SOFA: Sequential Organ Failure Assessment ${ }^{[13]}$

\begin{tabular}{|c|c|c|c|c|c|}
\hline \multirow{2}{*}{ Sistema } & \multicolumn{5}{|c|}{ SCORE } \\
\hline & 0 & 1 & 2 & 3 & 4 \\
\hline \multicolumn{6}{|l|}{ Respiración } \\
\hline $\mathrm{PaO}_{2} / \mathrm{FiO}_{2}^{\mathrm{a}, \mathrm{b}}(\mathrm{mmHg})$ & $\geq 400$ & $<400$ & $<300$ & $\begin{array}{l}<200 \text { con soporte } \\
\text { respiratorio }\end{array}$ & $\begin{array}{l}<100 \text { con soporte } \\
\text { respiratorio }\end{array}$ \\
\hline \multicolumn{6}{|l|}{ Coagulación } \\
\hline Plaquetas $\left(10^{3} / \mu \mathrm{l}\right)$ & $\geq 150$ & $<150$ & $<100$ & $<50$ & $<20$ \\
\hline \multicolumn{6}{|l|}{ Hígado } \\
\hline Bilirrubinas (mg/dl) & $<1,2$ & $1,2-1,9$ & $2,0-5,9$ & $6,0-11,9$ & $>12,0$ \\
\hline \multicolumn{6}{|l|}{ Cardiovascular } \\
\hline PAM o su manejo & $\begin{array}{c}\mathrm{PAM} \geq 70 \\
\mathrm{mmHg}\end{array}$ & $\begin{array}{c}\mathrm{PAM}<70 \\
\mathrm{mmHg}\end{array}$ & $\begin{array}{c}\text { Dopamina }<5 \\
\text { o dobutamina } \\
\text { (cualquier dosis)* }\end{array}$ & $\begin{array}{c}\text { Dopamina } 5,1-15 \\
\text { o epinefrina } \leq 0,10 \\
\text { norepinefrina } \leq 0,1^{*}\end{array}$ & $\begin{array}{c}\text { Dopamina }>150 \\
\text { epinefrina }>0,10 \\
\text { norepinefrina }>0,1^{*}\end{array}$ \\
\hline \multicolumn{6}{|l|}{ Sistema Nervioso Central } \\
\hline Escala de coma de Glasgow & 15 & $13-14$ & $10-12$ & $6-9$ & $<6$ \\
\hline \multicolumn{6}{|l|}{ Renal } \\
\hline Creatinina (mg/dL) & 1,2 & $1,2-1,9$ & $2,0-3,4$ & $3,5-4,9$ & $>5,0$ \\
\hline Gasto urinario (mL/día) & & & & $<500$ & $<200$ \\
\hline
\end{tabular}

$\mathrm{PaO}_{2}$ : Presión arterial de oxígeno FiO : Fracción inspirada de oxígeno PAM Presión arterial media

*Dosis de catecolaminas se dan en $\mu \mathrm{g} / \mathrm{kg} / \mathrm{min}$ por lo menos 1 hora

Adaptado de Singer et al. ${ }^{[3]}$

\section{SEPSIS - 3}

Recientemente, el Grupo de Trabajo de las Definiciones de Sepsis (Sepsis Definitions Task Force) ha publicado el consenso SEPSIS- ${ }^{[13]}$ con las definiciones actualizadas de sepsis y shock séptico y dos reportes con evidencia para validar estas nuevas definiciones ${ }^{[14,15]}$.

El consenso define "sepsis" como "una disfunción orgánica potencialmente mortal causada por una respuesta disregulada del huésped a la infección" [13]. Esta nueva definición implica una respuesta no homeostática del huésped a la infección e incluye el concepto de disfunción orgánica, lo cual implica severidad, necesidad de diagnóstico y manejo precoz y convierte en superfluo el término "sepsis severa". El Grupo de Trabajo propone la puntuación SOFA (Sequential Organ Failure Assessment, por sus siglas en inglés), que incluye una serie de criterios clínicos, de laboratorio y de manejo (Tabla 1), se asume que la puntuación SOFA basal es CERO, en pacientes sin disfunción orgánica pre-existente, mientras que, para definir los criterios clínicos que identifican los pacientes infectados con sepsis, el Grupo de Trabajo recomienda emplear un cambio en la puntuación SOFA inicial de 2 puntos o más para representar la disfunción orgánica (Tabla 2).

Otro concepto que introduce este consenso es el qSOFA (quick SOFA, por sus siglas en inglés) que puede servir para considerar una posible infección en pacientes en quienes no se ha diagnosticada infección previamente, no requiere pruebas de laboratorio, se puede realizar de manera rápida y se puede utilizar para el tamizaje de pacientes en quienes se sospecha un cuadro de sepsis probable. Se sugiere que los criterios qSOFA pueden ser utilizados de manera inmediata por los clínicos para evaluar la disfunción de órganos, para iniciar o intensificar la terapia en su caso, y para considerar la derivación a la atención crítica o aumentar la frecuencia de seguimiento, si aún no lo han llevado a cabo este tipo de acciones (Tabla 3).

El artículo de Seymour CW et al. ${ }^{[14]}$ describe que dentro de las UCls la validez predictiva de la mortalidad hospitalaria de la puntuación SOFA fue significativamente mejor que los criterios de SIRS y la puntación del qSOFA, pero, este análisis se realizó en pacientes en los que se sospechaba infección. Este mismo artículo concluye que en pacientes fuera de la UCI, la validez predictiva de la puntuación qSOFA para la mortalidad hospitalaria fue estadísticamente mayor que los criterios de SIRS y la puntuación SOFA.

Este Grupo de Trabajo también define "shock séptico" como "una subcategoría de la sepsis en la que las alteraciones circulatorias y del metabolismo celular son lo suficientemente profundas como para aumentar considerablemente la mortalidad" [33], proponiendo que los criterios para definir la ocurrencia de shock séptico: hipotensión, requerimiento sostenido de vasopresores para mantener una presión arterial media (PAM) $\geq 65 \mathrm{mmHg}$ y un nivel de lactato sérico mayor de $2 \mathrm{mmol} / \mathrm{L}$. El artículo de Shankar-Hari M et al. ${ }^{[15]}$ describe la

Tabla 3. Criterios de quick SOFA (qSOFA)

\section{Quick SOFA (qSOFA)}

Frecuencia respiratoria $\geq 22 \mathrm{resp} / \mathrm{min}$

Alteración del sensorio

Presión arterial sistólica $\leq 100 \mathrm{mmHg}$

Adaptado de Singer et al. ${ }^{[13]}$ 
metodología utilizada para la definición y criterios de shock séptico, concluyendo que la mejor definición es la presencia de hipotensión, requerimiento sostenido de vasopresores para mantener una presión arterial media (PAM) $\geq 65 \mathrm{mmHg}$ y un nivel de lactato sérico menor de $2 \mathrm{mmol} / \mathrm{L}$. Sin embargo, en el análisis sólo se consideraron estas tres variables, excluyéndose otras como marcadores de perfusión, trastorno del sensorio o biomarcadores. Por otro lado, debe considerarse para la aplicación de estos criterios la disponibilidad de la medición de lactato.

\section{LIMITACIONES}

Es importante entender esta nueva propuesta del nuevo consenso SEPSIS-3 nos brinda una definición que incluye los nuevos conceptos de la fisiopatología del "síndrome de sepsis", comenzaremos por resaltar las limitaciones planteadas por los autores:

La validación de estos criterios ha sido en base a cohortes retrospectivas, razón por la cual, plantean la necesidad de realizar estudios prospectivos para validar los nuevos criterios diagnósticos propuestos, el uso de la escala SOFA para diagnóstico de sepsis y la escala de puntuación qSOFA como tamizaje.

Otra limitante también subrayada por los autores del consenso, es el uso de la hiperlactatemia como criterio diagnóstico de shock séptico, ya que por un lado los niveles elevados de lactato se consideran un marcador importante de "shock críptico" en ausencia de hipotensión y se plantea la preocupación por la especificidad y su no disponibilidad de dosaje en todos los entornos, lo cual dificultaría el diagnóstico de shock séptico por los criterios que plantean. En este caso, cuando no se dispone de la medición del lactato, sería necesario emplear la hipotensión y otros criterios compatibles con hipoperfusión tisular para el diagnóstico de shock séptico.

Las puntuaciones SOFA y qSOFA se han evaluado y validado como predictores de mortalidad, pero no como criterios diagnósticos de sepsis, y no se ha evaluado la especificidad y sensibilidad de estas puntuaciones para definir un cuadro de sepsis.

Si bien la nueva definición de sepsis implica la inclusión de la respuesta del huésped a una infección, ninguno de los criterios incluye criterios de infección o la demostración microbiológica, puntos que aún siguen siendo difíciles de demostrar, las definiciones sólo se refieren a disfunción orgánica y severidad de la misma.

Los nuevos criterios diagnósticos en SEPSIS-3 para definir sepsis y shock séptico plantean dejar de lado el concepto de SIRS y reemplazarlo por la puntuación SOFA y qSOFA, sin embargo, la definición sigue siendo subjetiva. La definición utilizada tradicionalmente como un proceso inflamatorio asociado a una infección permite sospechar tempranamente que el paciente es portador de un cuadro de sepsis y no deberían abandonarse aún, por las definiciones planteadas en SEPSIS-3, ya que éstas no han demostrado mayor sensibilidad para el diagnóstico precoz.

La puntuación SOFA si bien es un método simple y efectivo para describir disfunción orgánica en pacientes críticamente enfermos y evaluar su evolución durante la permanencia de los pacientes en las UCls, no permite distinguir entre disfunción orgánica aguda, crónica o crónica reagudizada, ni permite determinar si la disfunción orgánica es secundaria a la ocurrencia de un cuadro infeccioso u otra condición que conlleve a esta falla orgánica.

La escala de puntuación SOFA ha sido descrita y validada en UCls, y para la realización de este consenso, se encontró que en las UCls la validez predictiva de la mortalidad hospitalaria de la puntuación SOFA fue estadísticamente mejor que la de los criterios de SIRS y la puntación del qSOFA, pero, en pacientes en los que se sospechaba infección.

Por las características de sus criterios, la escala de puntuación SOFA, que incluye exámenes de laboratorio, uso de ventilación mecánica e inotrópicos, no es un criterio diagnóstico que pueda ser aplicado en todos los niveles de atención y en salas de emergencia u hospitalización. En este contexto, debemos preguntarnos si estos criterios son conocidos por médicos generales y médicos de familia que se encuentran en los centros de atención primaria y hospitales de I y II nivel, a pesar que la escala de puntuación SOFA es fácil de aplicar, requiere difusión, capacitación de los médicos y sobretodo disponibilidad de los exámenes que se requieren para realizar la evaluación.

La alteración del estado mental, establecida en el q-SOFA como criterio, es inespecífica, debido a que puede ocurrir en otras condiciones médicas como encefalopatías metabólicas, trastornos hidroelectrolíticos, eventos vasculares, entre otros. Además, es importante tener en cuenta la ambigüedad asociada a la subjetividad en su evaluación, dado que no se ha establecido un punto de corte o una escala para la valoración de este criterio.

Además de lo mencionado, debemos tener en cuenta un aspecto relacionado con lo que se denominaría de "validez externa", y tiene que ver con el hecho de que la validación de las escalas de puntuación se realizó en una cohorte retrospectiva, de adultos con edad mayor o igual a 18 años con sospecha de infección, procedentes de hospitales comunitarios y académicos de Estados Unidos y un hospital de Alemania. Por lo tanto, antes de aplicarlos deberíamos preguntarnos si los resultados son válidos y extrapolables y aplicables a otros países, o a personas en quienes no se sospecha infección. Por lo tanto, es razonable, sugerir que antes de su aplicación se realicen estudios prospectivos en diferentes países y escenarios, para demostrar la aplicabilidad y validez de las escalas de puntuación propuestas tanto para el tamizaje como para el diagnóstico de sepsis. 


\section{DISCUSIÓN}

Como se pude apreciar, estamos asistiendo a un cambio de paradigma en torno a las definiciones para el diagnóstico de sepsis desde una clasificación de criterios basada en hallazgos clínicos, a una basada en fisiopatología. Si bien la nueva clasificación propuesta, pretende tener correlación con pronóstico y recoger el efecto de la sepsis en la homeostasis del organismo, pretendiendo una definición clara que pueda ser utilizada en la práctica clínica e investigación. Sin embargo, se abre un nuevo debate que obliga al desarrollo de investigación que permita validar los criterios propuestos, considerando que los pacientes con sepsis acuden a servicios médicos de atención primaria, emergencia, salas de hospitalización y UCls, y que es una patología que requiere de una temprana sospecha diagnóstica para administrar un tratamiento rápido, oportuno y pertinente, con criterios clínicos y de laboratorio de fácil acceso.

Finalmente, debemos aún aceptar que los criterios diagnósticos basados en el diagnóstico de SIRS, si bien presentan una serie de limitaciones, tienen una gran virtud y es la de permitir la temprana sospecha de sepsis, algo que aún no sabemos si ocurrirá con relación a estos nuevos criterios propuestos y de otro lado, se espera sea superada la limitación existente en cuanto a pronóstico de muerte de las definiciones, lo que es también incierto. Entonces, en este escenario, tal vez sería prudente mantener ambos criterios en la práctica clínica hasta tener las correspondientes validaciones y la confirmación de que podemos sustentar el cambio de los antiguos criterios con la tan necesaria evidencia.

Fuente de financiamiento:

Los autores declaran no haber recibido ninguna financiación para la realización de este trabajo.

\section{Declaración de conflicto de intereses:}

Los autores declaran no tener conflicto de intereses con la publicación de este artículo.

\section{REFERENCIAS BIBLIOGRÁFICAS}

1. Majno $G$. The ancient riddle of sigma eta psi iota sigma (sepsis). J Infect Dis. 1991;163(5):937-45.

2. American College of Chest Physicians/Society of Critical Care Medicine Consensus Conference: definitions for sepsis and organ failure and guidelines for the use of innovative therapies in sepsis. Crit Care Med. 1992;20(6):864-74.

3. Angus DC, van der Poll T. Severe sepsis and septic shock. N Engl J Med. 2013;369(9):840-51.

4. Wiersinga WJ, Leopold SJ, Cranendonk DR, van der Poll T. Host innate immune responses to sepsis. Virulence. 2014;5(1):36-44.

5. Levy MM, Fink MO, Marshall JC, Abraham E, Angus D, Cook D, et al. 2001 SCCM/ESICM/ACCP/ATS/SIS International Sepsis Definitions Conference. Intensive Care Med. 2003;29(4):530-8.

6. Sprung CL, SakrY, Vincent JL, Le Gall JR, Reinhart K, Ranieri VM, et al. An evaluation of systemic inflammatory response syndrome signs in the Sepsis Occurrence In Acutely III Patients (SOAP) study. Intensive Care Med. 2006;3(3)2:421-7.

7. Lai NA, Kruger P. The predictive ability of a weighted systemic inflammatory response syndrome score for microbiologically confirmed-infection in hospitalised patients with suspected sepsis. Crit Care Resusc. 2011;13(3):146-50.

8. Jaimes F, Garcés J, Cuervo J, Ramírez F, Ramírez J, Vargas A, et al. The systemic inflammatory response syndrome (SIRS) to identify infected patients in the emergency room. Intensive Care Med. 2003;29(8):1368-71.

9. Vincent JL. Dear SIRS, I'm sorry to say that I don't like you. Crit Care Med. 1997;25(2):372-4.

10 Vincent JL, Opal SM, Marshall JC, Tracey KJ. Sepsis definitions: time for change. Lancet. 2013;381(9868):774-5.

11. Kaukonen KM, BaileyM, Pilcher D, Cooper DJ, Bellomo R. Systemic inflammatory response syndrome criteria in defining severe sepsis. N Engl J Med. 2015;372(17):1629-38.

12. Czura CJ. "Merinoff Symposium 2010: sepsis"-speaking with one voice. Mol Med. 2011;17(1-2):2-3.

13. Singer M, Deutschman CS, Seymour CW, Shankar-Hari M, Annane $D$, Baver $M$, et al. The Third International Consensus Definitions for Sepsis and Septic Shock (Sepsis-3). JAMA. 2016;315(8):801-10.

14. Seymour CW, LiuVX, IwashynaTJ, Brunkhorst FM, ReaTD, Scherag $A$, et al. Assessment of clinical criteria for sepsis: for the Third International Consensus Definitions for Sepsis and Septic Shock (Sepsis-3). JAMA. 2016;315(8):762-74.

15. Shankar-Hari M, Phillips GS, Levy ML, Seymour CW, Liu VX Deutschman CS, et al. Developing a new definition and assessing new clinical criteria for septic shock: for the Third International Consensus Definitions for Sepsis and Septic Shock (Sepsis-3). JAMA. 2016;315(8):775-87. 